\title{
Epidemiology of spinal cord injuries in Novosibirsk, Russia
}

\author{
B Silberstein $^{1}$ and S Rabinovich ${ }^{2}$ \\ ${ }^{1}$ Department of Spinal Cord Injuries and ${ }^{2}$ Department of Neurosurgery, Research Institute of Traumatology and \\ Orthopaedics, 17 Frunze Street, 630091 Novosibirsk, Russia
}

\begin{abstract}
The incidence of spinal cord injuries (SCI) in Novosibirsk is 29.7 per million per year. Almost all of these SCI patients $(94.3 \%)$ are hospitalized to our clinic. Over the past 5 years (since 1989), a total of 196 patients with SCI were admitted. SCI were distributed as follows: cervical, 96 patients $(49.0 \%)$; thoracic, 54 (27.5\%); and lumbar, $46(23.5 \%)$. SCI was diagnosed using computed tomography (CT), magnetic resonance imaging (MRI), pneumomyelography, epidurography, radiography and electrophysiological methods (neuromyography, evoked potentials). All patients had a neurological deficit of varying degree; Frankel grade A, 64 patients $(32.7 \%) ; \mathrm{B}, 22(11.2 \%) ; \mathrm{C}, 67(34.2 \%)$; and D, 43 $(21.9 \%)$. Almost $40 \%(39.7 \%)$ of the patients had unstable fractures, most of them being in the cervical spine. One hundred and ninety patients were operated on, 52 within $3-4 \mathrm{~h}$ after trauma. Several types of operation were used: anterior decompression (106 patients); posterior decompression (64 patients); omentomyelopexia (seven patients); meningomyeloradiculolysis (13 patients). Conservative treatment ('halo' traction) was applied in six patients. No patient was made worse because of the surgery. Twenty four patients had a complete neurological recovery, 113 patients could be reclassified into a higher group (Frankel classification), and 59 patients had no neurological improvement. The overall mortality was $16.8 \%$ (13.7 postoperatively).
\end{abstract}

Keywords: spinal cord injury; epidemiology; incidence; Novosibirsk; Russia

\section{Introduction}

As has been shown by Western doctors, establishment of a regional spinal cord injury care system is the most effective way of managing patients with SCI. ${ }^{1}$ We attempted to organize such a system in Novosibirsk, and since 1989 all patients who had a SCI within the territory of Novosibirsk have been treated in the Department of Spinal Cord Injuries, Research Institute of Traumatology. Our 5 year experience in the diagnosis and treatment of these patients as well as epidemiological data obtained during this period provide the basis for this study.

\section{Materials and methods}

We analysed all inpatients with SCI during 5 consecutive years from 1989 to 1993 . Those with an extradural nerve root or a plexus injury were not included. SCI was diagnosed using CT, MRI, pneumomyelography, epidurography, radiography and electrophysiological methods (neuromyography, evoked potentials). The neurological deficit was classified according to the Frankel classification. ${ }^{2}$ One hundred and ninety patients had an operation, and the types of operation used were as follows: anterior decompression, posterior decompression, omentomyelopexia, and meningomyeloradiculolysis. Six patients had conservative treat- ment ('halo' traction). As a well equipped rehabilitation centre is not available in Novosibirsk, we also provided rehabilitation for these patients using physical therapy and medication.

\section{Results}

Incidence

Over the past 5 years, 196 SCI patients were hospitalized to our clinic, and 12 to other clinics in Novosibirsk (they are not included in this study). As the population of Novosibirsk is 1.4 million (according to the data of 1 January 1994), the incidence of SCI is 29.7 per million per year (Table 1). All patients had a neurological deficit of varying degree (Table 2 ).

\section{Gender and age}

There were $153(78.1 \%)$ males and $43(21.9 \%)$ females. The number of males was 3.5 times greater than the females. The mean age was 34.7 years in the males and 32.3 in the females. The age distribution showed a peak in the age group of 20-29 years. The incidence of SCI also slightly increased at the age of 50-59 years, whereas in patients from the other age groups it was minimal (Figure 1). 
Table 1 Incidence of SCI in Novosibirsk

\begin{tabular}{lc}
\hline Population (million) & 1.4 \\
Number of patients hospitalised to our clinic & 196 \\
Number of patients hospitalised to other clinics & 12 \\
Total & 208 \\
Incidence (per million) & 29.7 \\
\hline
\end{tabular}

Table 2 Neurological status of hospitalised patients (on admission)

\begin{tabular}{lrl}
\hline Frankel grade A & 64 & $(32.7 \%)$ \\
Frankel grade B & 22 & $(11.2 \%)$ \\
Frankel grade C & 67 & $(34.2 \%)$ \\
Frankel grade D & 43 & $(21.9 \%)$ \\
Total & 196 & $(100 \%)$ \\
\hline
\end{tabular}

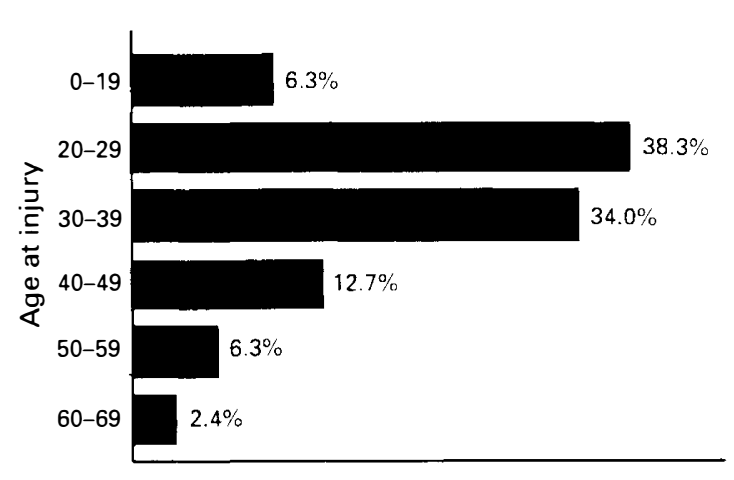

Percentage of patients

Figure 1 Age distribution at the time of injury

\section{Causes of injuries}

Traffic accidents and falls from a height were the most common causes of SCI. Diving was also a common cause, especially in younger patients. Two patients were stabbed, and one had a shotgun injury. Gymnastics and wrestling were the causes of sport injuries in $9.7 \%$ patients (Table 3 ). It is of interest that $20.3 \%$ of males and $9.3 \%$ of females were found to be under the influence of alcohol at the time of their injury.

\section{Characteristic of spine injuries}

There were several types of vertebral column lesions (Table 4): wedge-shaped fractures and fracturedislocations were noted to be commonest, burst fractures and isolated fractures of pedicles or articular processes were less often seen.

As is shown in Fable 5, the most frequent localisation of a spinal injury was of the cervical spine $(49 \%)$, with C3-7 being 5.4 times more frequent than $\mathrm{C} 1-2$. The relationship between the level of injury and the cause of the trauma is indicated in Table 6.

According to the Holdsworth classification, ${ }^{3}$ all spinal injuries were divided into two groups-stable fractures and unstable fractures. Unstable fractures occurred more frequently in cervical spine $(64 \%$ of all
Table 3 Causes of SCI in Novosibirsk

\begin{tabular}{lrr}
\hline Fall from a height & & \\
$\quad$ Life trauma (fall from a tree, & 38 & $19.4 \%$ \\
$\quad$ balcony, roof, etc) & & \\
Industrial accident & 21 & $10.7 \%$ \\
Escape from chasers & 14 & $7.2 \%$ \\
Suicide & 6 & $3.1 \%$ \\
$\quad$ Total & 79 & $40.4 \%$ \\
Traffic accident & & \\
Car, truck & 19 & $9.7 \%$ \\
Pedestrian & 15 & $7.7 \%$ \\
Motorbike & 9 & $4.6 \%$ \\
Bicycle & 6 & $3.1 \%$ \\
$\quad$ Total & 49 & $25.1 \%$ \\
Diving & 46 & $23.2 \%$ \\
Sport & 19 & $9.7 \%$ \\
Gunshot, stab & 3 & $1.6 \%$ \\
Total & 196 & $100 \%$ \\
\hline
\end{tabular}

Table 4 Type of spinal lesion followed by SCI

\begin{tabular}{lrr}
\hline Fracture-dislocations & 78 & $39.7 \%$ \\
Wedge-shaped fractures & 75 & $38.3 \%$ \\
Burst fractures & 35 & $17.9 \%$ \\
Isolated fractures of pedicles and & 8 & $4.1 \%$ \\
articular processes & & \\
Total & 196 & $100 \%$ \\
\hline
\end{tabular}

Table 5 Level of spinal lesion resulting from SCI

\begin{tabular}{lcrr}
\hline Cervical spine & C1-2 & 15 & $7.7 \%$ \\
& C $3-7$ & 81 & $41.3 \%$ \\
Thoracic spine & T1-12 & 54 & $27.5 \%$ \\
Lumbar spine & L1-5 & 46 & $23.5 \%$ \\
Total & & 196 & $100.0 \%$ \\
\hline
\end{tabular}

Table 6 Causes of trauma and the level of the spinal injury

\begin{tabular}{lrr}
\hline Cervical spine & & \\
Diving & 46 & $23.5 \%$ \\
Fall from a height & 21 & $10.7 \%$ \\
Traffic accident & 19 & $9.7 \%$ \\
Sport & 8 & $4.1 \%$ \\
Stab & 2 & $1.0 \%$ \\
Total & 96 & $49 \%$ \\
Thoracic spine & & \\
Fall from a height & 23 & $11.7 \%$ \\
Traffic accident & 19 & $9.7 \%$ \\
Sport & 11 & $5.6 \%$ \\
Gunshot & 1 & $0.5 \%$ \\
Total & 54 & $27.5 \%$ \\
Lumbar spine & & \\
$\quad$ Fall from a height & 35 & $17.9 \%$ \\
Traffic accident & 11 & $5.6 \%$ \\
Total & 46 & $23.5 \%$ \\
Total & 196 & $100 \%$ \\
\hline
\end{tabular}


unstable fractures) and predominated among the cervical spine injuries (Figure 2).

\section{Treatment}

As our clinic is available for operations $24 \mathrm{~h}$ a day, the interval between the occurrence of trauma and the admittance to the hospital was usually quite short, and most patients $(52 \%)$ were operated on within $2-8 \mathrm{~h}$ of trauma (Figure 3). Patients with an acute SCI who had surgery later had been transferred from other emergency hospitals. We also hospitalised some patients with a spinal fracture intreated for more than a year after the SCI in order to perform reconstructive surgery.

One hundred and ninety patients were operated on, 52 of them within 3-4 h after trauma. Several types of operation were used: anterior decompression (106 patients), posterior decompression (64 patients), omentomyelopexia (seven patients), meningomyeloradiculolysis (13 patients). Six patients were managed conservatively with 'halo' traction.

No patient was made worse as a result of surgery. Twenty four patients made a complete recovery, 113 patients could be reclassified into a higher group (Frankel classification), and 59 patients had no neurological improvement. The overall mortality was $16.8 \%$ (13.7 postoperatively). Most patients who died $(84.8 \%)$ had injuries of the cervical spinal cord at the level of $\mathrm{C} 4$ and above.

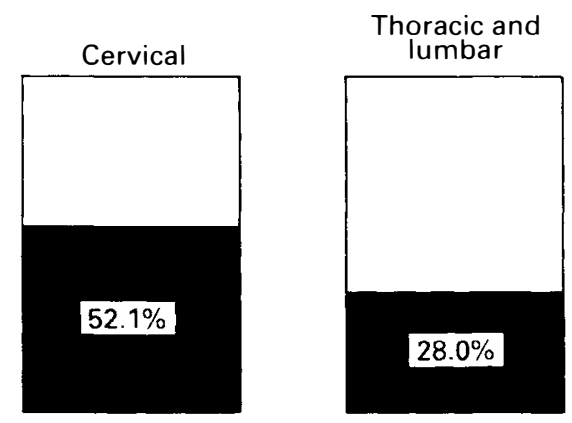

Figure 2 Frequency of unstable lesions of spine

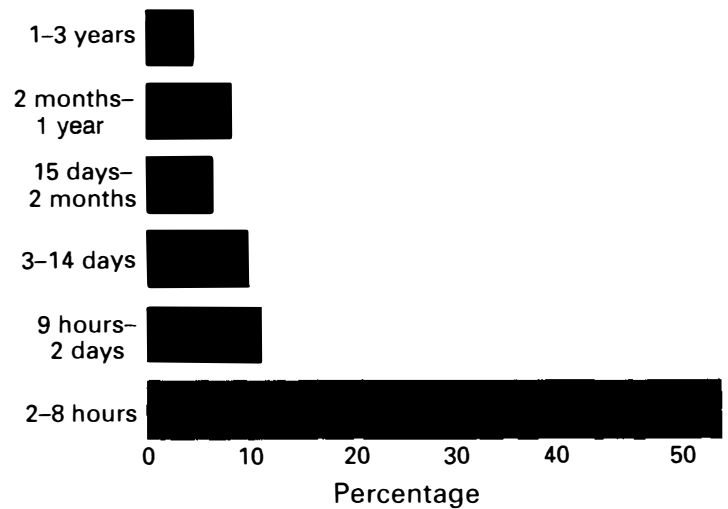

Figure 3 Time between the occurence of trauma and surgical treatment

\section{Discussion}

It is unquestionable that truly comprehensive medical care for SCI patients can only be provided by specialist centres. The Department of Spine and Spinal Cord Injuries was founded in 1989 , and since that time all patients who had suffered such trauma in Novosibirsk have been hospitalized in our clinic. We undertook a study of the epidemiology of SCI in order to estimate the results of our work, plan measures for the improvement of medical care in the future (the number of beds required, expenses, etc) as well as to establish ways of preventing SCI.

The incidence of SCI in Novosibirsk is comparable to that in other countries. ${ }^{4}$ Although it is slightly lower than in Florida, FRG or Japan, this difference can be due to the relatively small population (1.4 million) and the number of patients analysed in this study. The male/female ratio is $3.5: 1$, and this is also a common finding. ${ }^{4}$

One of the characteristic features of SCI in Novosibirsk is the low mean age of the patients $(34.7$ years in males and 32.2 years in females, compared to 48.3 years and 49.2 years in Japan, respectively). This fact can be understood if the distinction between the causes of injuries in other regions is taken into accout. The high incidence of SCI due to diving into shallow water $(23.2 \%)$ and sport injuries $(9.7 \%)$ which are typical for younger people may be an explanation.

Another feature that distinguishes our study from others is that the most frequent cause of injury was a fall from a height, while in other reports traffic accidents have been predominant. There are several reasons for this discrepancy. Firstly, traffic in Russia has some peculiarities (speed limit $60 \mathrm{~km} \mathrm{~h}^{-1}$, absence of motorways, etc); also, the number of cars per person in Russia is significantly lower than in other countries. On the other hand the above-mentioned features (high incidence of SCI due to diving and sports) can also contribute to this discrepancy. Finally, as was shown by Japanese investigators, elderly people are often injured in traffic accidents, which are not very common in Russia. The point is that very few people at this age drive a car; also, life expectancy in Russia is less than that it is in Japan.

Regarding the mortality rate in our series of patients with SCI of the cervical spine, it is comparable to data reported from around the world. ${ }^{5}$ However, the overall mortality in SCI patients treated in our clinic is slightly higher than that reported from other countries. ${ }^{6}$ There are at least two factors which may contribute to this fact, the first being that our clinic is the only hospital in Novosibirsk which provides comprehensive medical care for SCI patients. Therefore, we admit a high proportion of patients who had been previously hospitalized in other clinics and had received inadequate treatment.

We also consider that our concept (according to which all patients who do not have a contraindication to surgery should, as soon as possible, undergo operative decompression of the spinal cord and stabilisation of the injured part of the spine) may also influence our 
mortality rate. During the past few years it has been shown that operation itself is not likely to improve the neurological abnormalities, and the value of surgical intervention is disputable. ${ }^{6-12}$ This approach can certainly be justified, but only on the condition that a well-organised rehabilitation system is provided. Unfortunately, the facilities which are necessary to enable a patient to have prolonged bed rest during the time required for consolidation and stabilization of spine at the level of injury are not available in our clinic, and the percentage of complications (pressure sores, infections of respiratory and urinary tract) in conservatively treated patients is very high. As a consequence, the results of conservative treatment are unsatisfactory, and the mortality rate considerably exceeds that in the patients who had operative treatment. This is the reason why we consider the wide use of surgery in SCI patients to be warranted.

Other data obtained in our study are consistent with results previously published. ${ }^{4}$ In particular, the most severe spine lesions involved the cervical spine, and more than $80 \%$ of the lesions of the spine were unstable. Concerning lesions of the thoracic and lumbar spine, fractures at the level of T12-L1 were the most frequent. ${ }^{13}$

This study of the epidemiology of SCI in Novosibirsk was the first attempt to carry out an epidemiological survey in the Siberian region. As the number of SCI patients hospitalised in our clinic is constantly growing and we started to provide medical care for SCI patients not only from Novosibirsk, but also from the Novosibirsk region (1.8 million), the epidemiological data are becoming much more important. Therefore, the Department of Epidemiology of SCI and our department are now carrying out a new, more comprehensive epidemiological study.

Analysis of the causes of SCI injuries, and results of treatment (which are not promising to far), clearly show that the importance of the prevention of SCI, especially in younger patients, must be emphasized. A campaign for the prevention of SCI which has been started in Japan should also be organized in Russia. We should be happy to collaborate with our Japanese colleagues in this field.

\section{References}

1 Hasegava $\mathrm{Y}$ et al. Spinal cord injury in a rehabilitation hospital in Japan. Paraplegia 1994; 32: 47-51.

2 Frankel HL et al. The value of postural reduction in the initial management of closed injuries of the spine with paraplegia and tetraplegia. Paraplegia 1969; 7: 179-192.

3 Holdsworth FW. Fractures, dislocations and fracture-dislocations of the spine. J Bone Joint Surg Br 1963; 45: 6-20.

4 Shingu H, Ikata T, Katoh S, Akatsu T. Spinal cord injuries in Japan: a nationwide epidemiological survey in 1990. Paraplegia 1994; 32: 3-8.

5 Watson Payne Roye Jr et al. Cervical spine cord injury-a public catastrophe. J Trauma 1988; 8: 1260-1264.

6 Tator $\mathrm{CH}$ et al. Comparison of surgical and conservative management in 208 patients with acute spinal cord injury. Le Journal Canadien Des Sciences Neurolgiques 1987; 14: 60-69.

7 Loembe PM. Traumatismes vertebro-medullars attitudes therapeutiques au Gabon. Acta Orthopedica Belgica 1991; 57: 31-42.

8 Alho A. Operative treatment as a part of comprehensive care for patients with injuries of the thoracolumbar spine. A review. Paraplegia 1994; 32: 509-516.

9 Stauffer ES. Neurologic recovery following injuries of the cervical spinal cord and nerve roots. Spine 9: 532-534.

10 Willen $G$ et al. Unstable thoracolumbar fractures. A comparative clinical study of conservative treatment and Harrington instrumentation. Spine 10: 111-122.

11 Woolsey RM. Rehabilitation outcome following spinal cord injury. Arch Neurol 1985; 42: 116-119.

12 Harris $\mathrm{P}$ et al. The prognosis of patients sustaining severe cervical spine injury (C2-C7 inclusive). Paraplegia 1980; 18: 324-330.

13 Titze A. Kyphozen nach wirbelbruchen. Orthopedics 1973; 23: 173-177. 